\title{
Fluorescence excitation spectra of jet-cooled 4-(diisopropylamino)benzonitrile and related compounds
}

\author{
Rainer Daum ${ }^{\text {a }}$, Sergey Druzhinin ${ }^{\mathrm{a}}$, Dietmar Ernst ${ }^{\mathrm{b}}$, Lutz Rupp ${ }^{\mathrm{b}}$, \\ Jörg Schroeder ${ }^{\mathrm{a}, \mathrm{b}, *}$, Klaas A. Zachariasse ${ }^{\mathrm{a}}$ \\ a Abteilung Spektroskopie und Photochemische Kinetik, Max-Planck-Institut für Biophysikalische Chemie, Am Fassberg, \\ D-37077 Göttingen, Germany \\ ${ }^{\mathrm{b}}$ Institut für Physikalische Chemie der Universität, Tammannstr. 6, D-37077 Göttingen, Germany
}

Received 2 February 2001; in final form 23 March 2001

\begin{abstract}
Fluorescence excitation spectra of 4-(diisopropylamino)benzonitrile (DIABN) and 4-(dimethylamino)benzonitrile (DMABN) in thermal vapour and seeded jet expansions are compared. The spectrum of jet-cooled DIABN shows an intense $0-0$-transition at $31751.8 \mathrm{~cm}^{-1}$. The spectrum collapses at excess excitation energies above $800 \mathrm{~cm}^{-1}$, indicating the presence of an efficient non-radiative decay channel. In the gas phase, fluorescence emission of DIABN occurs from the intramolecular charge transfer (ICT) state. The non-radiative decay channel, therefore, is attributed to rapid ICT in the isolated molecule. The related compounds 4-(methylamino)-3,5-dimethylbenzonitrile (MHD), 4-(azetidinyl)-3,5dimethylbenzonitrile (M4D), and 4-(dimethylamino)-3,5-dimethylbenzonitrile (MMD) in the jet show extremely weak and structureless emission. (c) 2001 Elsevier Science B.V. All rights reserved.
\end{abstract}

\section{Introduction}

The origin of the dual fluorescence of 4-(dimethylamino)benzonitrile (DMABN) and related compounds in solution continues to be the subject of detailed investigation and controversial discussion $[1,2]$. While there seems to remain little doubt that, following excitation of a locally excited state (LE), structural changes accompanied by intramolecular charge transfer (ICT) lead to population of a second emitting state, conflicting hypotheses concerning the nature of these structural rearrangements have been put forward. Among the

\footnotetext{
* Corresponding author. Fax: +49-551-201-1501.

E-mail address: jschroe@gwdg.de (J. Schroeder).
}

mechanisms discussed, the most prominent is the twisted intramolecular charge transfer (TICT) model as originally proposed by Grabowski et al. [3]. In essence it states that intramolecular charge separation is accompanied by a $90^{\circ}$ twist of the dimethylamino group with respect to the benzonitrile moiety, taking the molecule from a more or less planar conformation in the LE state to a perpendicular structure in the ICT state. There is substantial evidence, however, that alternative mechanisms have to be considered in order to account for kinetics and spectra observed for a variety of related compounds under different physical conditions, ranging from the isolated molecule to supercritical fluid, as well as in nonpolar and polar liquid solutions. In this context, we recently proposed a planar intramolecular 
charge transfer (PICT) model [2,4-6] which assumes an essentially planar conformation of the ICT state, whereas the LE state retains at least part of the pyramidal structure of the dimethylamino group present in the electronic ground state. In this PICT-model, the energy gap $\Delta E\left(\mathrm{~S}_{1}, \mathrm{~S}_{2}\right)$ between the two lowest excited singlet states is of crucial importance for the dynamics of ICT state formation. Its magnitude can be controlled either externally by varying solvent polarity or internally by appropriately modifying the molecular structure of the aminobenzonitriles. Systematic investigations of structural and solvent effects on ICT have therefore been made to elucidate the detailed mechanism of formation and the molecular nature of the emitting ICT state [1-6].

Recently, dual fluorescence of the DMABN derivative 4-(diisopropylamino)benzonitrile (DI$\mathrm{ABN}$ ) in alkane solvents was reported and shown to be associated with fast ICT [6], while it is wellknown that DMABN itself does not exhibit dual fluorescence in such non-polar solvents. This observation lends strong support to the PICT model, as fast ICT in DIABN is favoured by the comparatively small intrinsic energy gap $\Delta E\left(\mathrm{~S}_{1}, \mathrm{~S}_{2}\right)$. Moreover, the bulkiness of the isopropyl substituents in DIABN reduces the pyramidality of the amino nitrogen [6] and should retard any twisting motion of the amino group. ICT with DMABN in a non-polar environment is prevented by a much larger $\Delta E\left(\mathrm{~S}_{1}, \mathrm{~S}_{2}\right)$ gap than that present in DIABN.

Under collision-free conditions in a supersonic jet expansion, isolated DMABN appears to emit only from the LE state [7-9] with a lifetime around $5 \mathrm{~ns}$ independent of excitation energy in the energy range up to about $900 \mathrm{~cm}^{-1}$ above the $0-0$-transition $[8,10]$. In this region, the laser induced fluorescence (LIF) excitation spectrum shows no indications for electronic coupling between the emitting $\operatorname{LE}\left(\mathrm{S}_{1}\right)$ and the higher lying $\mathrm{S}_{2}$ state. Based on results of a careful investigation of rotational contours of some prominent vibronic transitions in DMABN [11], however, it has been argued recently that already at low excess energy $E=h v_{\mathrm{exc}}-E_{0}^{0}\left(v_{\mathrm{exc}}\right.$ is the frequency of the exciting laser pulse and $E_{0}^{0}$ the energy of the $0-0$-transition) there is evidence for substantial coupling between electronic states. For smaller $\Delta E\left(\mathrm{~S}_{1}, \mathrm{~S}_{2}\right)$ energy gaps such as in DIABN, one would expect that even stronger perturbations indicative of enhanced $\mathrm{S}_{1}-\mathrm{S}_{2}$ coupling appear in the LIF excitation spectrum at sufficiently high excess energy. Such effects were inferred, e.g., for jet-cooled isolated 4pyrrolidinobenzonitrile from the change of shape of the fluorescence emission spectrum upon excitation at $773.1 \mathrm{~cm}^{-1}$ excess energy [12] and the methyl esters of 4-(pyrrolidinyl)benzoic acid (P5ME) and 4-(dimethylamino)benzoic acid based on the appearance of an underlying continuum component in the spectrum and characteristic changes in the rotational contour with increasing excitation energy $[12,13]$. The purpose of the present study is the comparison of LIF excitation spectra of jet-cooled DMABN derivatives having different $\Delta E\left(\mathrm{~S}_{1}, \mathrm{~S}_{2}\right)$ energy gaps. In addition to DIABN and DMABN we investigated jet expansions of 4-methylamino-3,5-dimethylbenzonitrile (MHD), 4-(azetidinyl)-3,5-dimethyl-benzonitrile (M4D), and 4-(dimethylamino)-3,5-dimethylbenzonitrile (MMD). The structures of the compounds investigated in this study are given below.

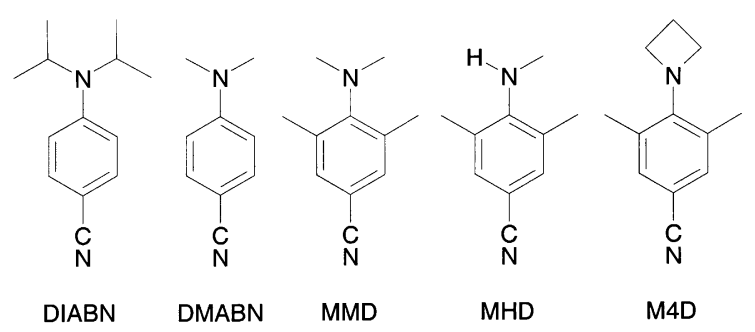

\section{Experimental}

Details of our experimental setup have been described elsewhere $[14,15]$. We therefore only summarize the main features. The jet apparatus consists of a vacuum chamber evacuated by a diffusion pump (Edwards Diffstak 250/2000M) backed by a rotary pump (Edwards E2M40). The samples were heated to $90-150^{\circ} \mathrm{C}$, entrained in 1.5-10 bar helium and expanded into the vacuum using a General Valve Series 9 pulsed nozzle. The nozzle orifice had a diameter of $100 \mu \mathrm{m}$.

For measuring fluorescence excitation spectra, the jet beam is crossed at right angles by a 
perpendicularly polarized laser beam. The laser system consists of a dye-laser (Lambda Physik Scanmate II, $0.15 \mathrm{~cm}^{-1}$ resolution) pumped by a Nd:YAG laser (Quantel Brilliant $\omega$ ) at a $10 \mathrm{~Hz}$ repetition rate. The dye laser beam was frequency doubled in a KDP crystal to generate excitation wavelengths in the range from 268 to $325 \mathrm{~nm}$. The broadband fluorescence was collected perpendicularly to the plane spanned by laser beam and jet expansion and focused by a collimating f/1 lens system onto a microchannel plate photomultiplier (MCP-PMT, Hamamatsu R3809U). The resulting signal was accumulated in a home-made boxcar integrator, digitized and stored in a computer.

Vapour phase absorption and fluorescence spectra were measured employing a Cary 500 Scan spectrophotometer and an ISA-SPEX Fluorolog 3-22 fluorometer, respectively. The samples were contained in a sealed quartz cuvette of $1 \mathrm{~cm}$ path length. Care was taken to maintain the optical windows at a higher temperature than the sample reservoir of the cell. Temperatures were controlled by a JUMO Dicon 1000 Universal Profile Controller. The vapour pressure of the compounds was adjusted by varying the temperature of the reservoir.

\section{Results and discussion}

\subsection{DMABN and DIABN}

In order to obtain for the class of compounds investigated here a relative standard for our specific experimental set-up, the fluorescence excitation spectrum of jet-cooled DMABN was measured, see Fig. 1, resulting in a spectrum similar to those already published in the literature [7-9,13,16,17]. The weak $0-0$-transition was found at $E_{0}^{0}=32246.5 \mathrm{~cm}^{-1}$, in excellent agreement with the value given by Salgado et al. [11]. As in dimethylaniline, the low frequency part $\left(0-250 \mathrm{~cm}^{-1}\right)$ of the LIF excitation spectrum is dominated by transitions arising from torsion of the methyl rotors of the dimethylamino substituent [18]. Though there still may remain some uncertainty regarding the detailed assignment and the coupling with the amino inversion mode

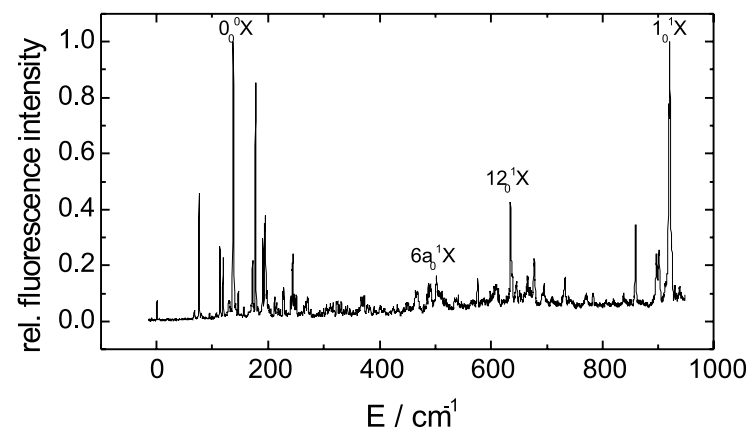

Fig. 1. LIF excitation spectrum of jet-cooled isolated DMABN. Pulsed helium expansion at 9 bar stagnation pressure, sample temperature $150^{\circ} \mathrm{C}$. The four main band systems of the frequency range are indicated at their most prominent transition, $X=137 \mathrm{~cm}^{-1}$.

$[16,19,20]$, following the arguments put forward in the case of dimethylaniline [18] it seems very reasonable to assume that the weak $0-0$-transition observed for DMABN is caused by a substantial change of the equilibrium torsion angle of the methyl rotors between the ground and the locally excited state. The low frequency pattern then repeats itself in combination transitions with 365, 498 , and $783 \mathrm{~cm}^{-1}$ that are assigned to (benzenelike notation [21]) modes 6a (in-plane ring deformation), 12 (in-plane ring stretch), and 1 (in-plane ring breathing), respectively [19].

Jet-cooled DIABN likewise exhibits a well-defined structured LIF excitation spectrum as shown in Fig. 2. From a comparison of the fluorescence

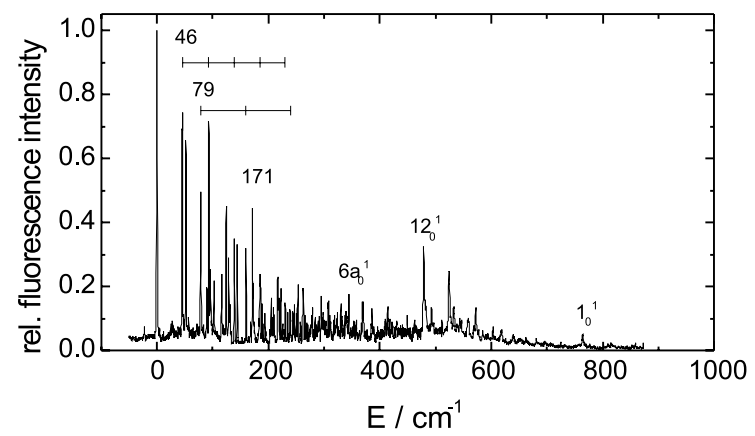

Fig. 2. LIF excitation spectrum of jet-cooled isolated DIABN. Pulsed helium expansion at 5 bar stagnation pressure, sample temperature $100^{\circ} \mathrm{C}$. The main band systems are labelled and two low frequency progressions are identified. 
intensities of corresponding spectral lines of DIABN and DMABN, a relative fluorescence quantum yield of about $10^{-2}$ is estimated for DIABN with respect to that of DMABN, assuming similar values for the absorbance of DMABN and DIABN under our experimental conditions. The lowest energy line in the DIABN spectrum was found to have the largest intensity, similar to LIF excitation spectra reported for 4-(diethylamino)benzonitrile [22], 4-(pyrrolidino)benzonitrile (P5C), and 4-(pyrrolidino)benzoic acid methyl ester [13], which indicates that only little structural change takes place in DIABN upon 0-0-excitation to the LE state. Equivalently, a strong 0-0-transition is found for $N, N$-diethylaniline, supporting the assumption that methyl rotor torsion is responsible for the low frequency part of the LIF spectrum [18]. Line positions are given with respect to the origin at $31751.8 \mathrm{~cm}^{-1}$. The red shift of the $0-0$-transition by $495 \mathrm{~cm}^{-1}$ compared to that of DMABN is also reflected in a corresponding red shift of the vapour phase absorption spectra between DMABN and DIABN as illustrated in Fig. 3.

Due to the presence of the two isopropyl groups, the LIF spectrum of DIABN is very crowded already at small excess energy and contains a number of intense lines indicating efficient excitation of the multitude of twisting modes present in this molecule. Two progressions may be identified, one with a $46 \mathrm{~cm}^{-1}$ mode that can be

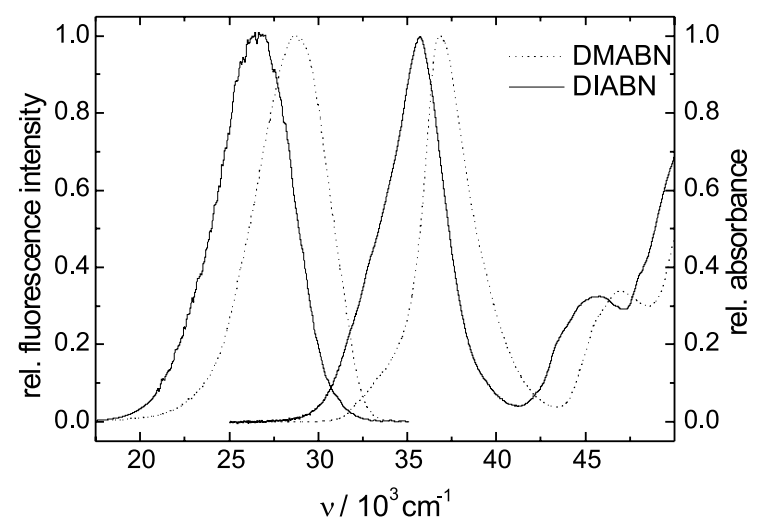

Fig. 3. Vapour phase absorption and fluorescence spectra excited at $280 \mathrm{~nm}$ of DMABN (dotted line; absorption at $150^{\circ} \mathrm{C}$ and fluorescence at $90^{\circ} \mathrm{C}$ ) and DIABN (full line; absorption at $100^{\circ} \mathrm{C}$ and fluorescence at $90^{\circ} \mathrm{C}$ ). followed up to $v=5$ at $230 \mathrm{~cm}^{-1}$, and another one with $79 \mathrm{~cm}^{-1}$ up to $v=3$ at $238 \mathrm{~cm}^{-1}$. There appear to be also a number of low frequency combination bands that are not further specified here. Similar to DMABN, the complex low frequency pattern reappears in combination with higher frequency modes at 171,344 , and $479 \mathrm{~cm}^{-1}$. The latter two may again be assigned to modes $6 \mathrm{a}$ and 12 as in the case of DMABN.

Another striking difference between the LIF spectra of DMABN and DIABN is the complete absence of intense combination bands with mode 1 . Only the weak origin of this band system is visible at $764 \mathrm{~cm}^{-1}$. The benzene-like modes $6 \mathrm{a}, 12$, and 1 are shifted to lower frequencies by about $20 \mathrm{~cm}^{-1}$ with respect to their positions in the LIF spectrum of DMABN, an effect that is in line with an equivalent shift between the spectra of $\mathrm{ABN}$ and DMABN [19]. As far as can be judged considering this congested spectrum, there appears to be very little background, indicating the absence of strong electronic coupling for energies $E \leqslant 600 \mathrm{~cm}^{-1}$. A similar conclusion has been drawn for DMABN and $\mathrm{P} 5 \mathrm{C}$ [13]. This lack of strong coupling is also born out by the widths of the rotational contours which are close to $1.3 \mathrm{~cm}^{-1}$. At higher excess energy, the intensity rapidly drops to very low values, with a corresponding significant increase in linewidth. Both observations suggest that an efficient non-radiative decay channel becomes accessible to the LE state in this energy range. In view of the large efficiency of the ICT reaction of DIABN in alkane solvents [6], we assign this non-radiative decay to the channel leading eventually to the ICT state in solution.

Strong additional evidence to support this hypothesis may be obtained from a comparison of the fluorescence emission spectra of DMABN and DIABN. In $n$-hexane solution [6], the Stokes shift between the absorption and fluorescence emission spectrum of DMABN is about $6500 \mathrm{~cm}^{-1}$, and, in this case, emission only takes place from the LE state. In contrast, the Stokes shift for DIABN is close to $8000 \mathrm{~cm}^{-1}$, and most of the emission is from the ICT state with a small contribution from the LE state which is visible as a small shoulder at the blue side of the main fluorescence band at about $29500 \mathrm{~cm}^{-1}$. The corresponding spectra in 
the vapour phase are shown in Fig. 3. For DMABN, the maximum of the absorption spectrum is shifted $1000 \mathrm{~cm}^{-1}$ to the blue, while that of the LE fluorescence spectrum changes to a much smaller extent. In the case of DIABN, both the absorption and the fluorescence emission band maximum roughly shift $1000 \mathrm{~cm}^{-1}$ to the blue, maintaining the same Stokes shift as in non-polar solution. The fluorescence peaks at about $26500 \mathrm{~cm}^{-1}$, which still is $3000 \mathrm{~cm}^{-1}$ to the red of the LE band in $n$-hexane solution, clearly indicating that also in the vapour phase the fluorescence of DIABN predominantly originates from the ICT state, whose emission band now completely obliterates the weak shoulder of the LE state emission.

\subsection{MMD, M4D and MHD}

Having taken LIF excitation spectra of DMABN and DIABN, we prepared corresponding expansions with MMD, M4D and MHD. The wavelength range $30 \mathrm{~nm}$ up and downward from the maximum of the respective bulk vapour phase absorption was scanned carefully. With MMD we obtained a very weak and broad LIF excitation band as described previously by two other groups $[17,23]$. The intensity of the fluorescence was about a factor of $10^{3}$ weaker than for DMABN and close to our detection limit. In the case of M4D the light intensity at the detector was even an order of magnitude less than that found with MMD and we were hence not able to identify an LIF excitation band. MHD gave signals similar in intensity to those obtained from MMD. Fig. 4 shows a combination of four spectral scans of the emission from a jet seeded with MHD (sample temperature $150^{\circ} \mathrm{C}$ ) corrected for pump laser intensity. The structure at short excitation wavelengths is due to etalon effects at wavelengths below $280 \mathrm{~nm}$ resulting from blocking filters. The signal-to-noise ratio is very poor, a result of the very low fluorescence intensity, similar to that observed in the case of MMD. The spectral shape and position of the broad excitation band of MHD may still be contaminated by stray light, with an intensity that varies with laser output power during the spectral scan. Therefore, we are reluctant to identify the

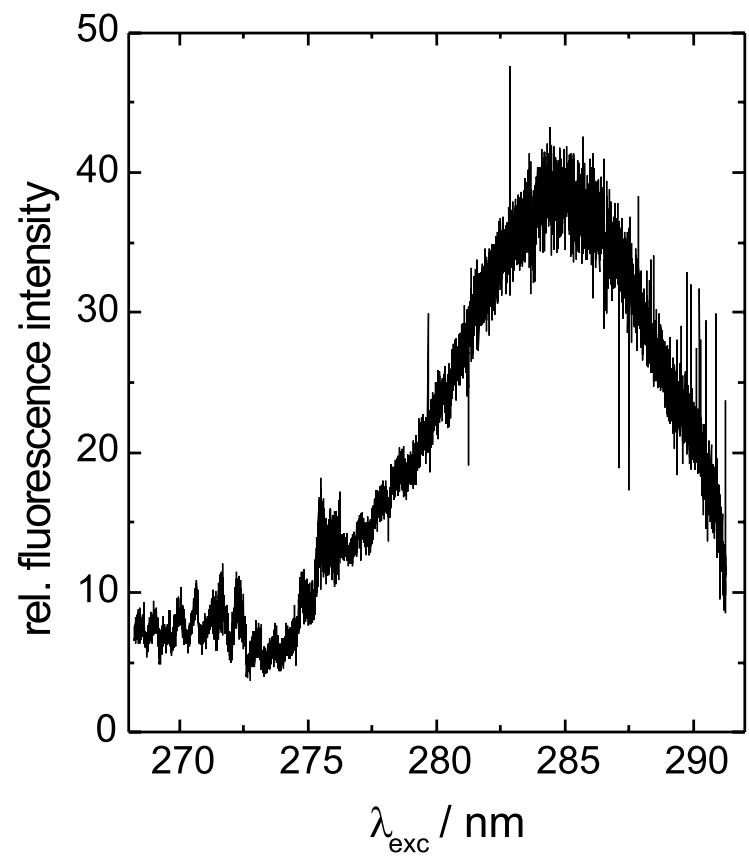

Fig. 4. Emission spectrum normalized to laser power variation obtained from an MHD-seeded helium jet (7 bar stagnation pressure; $120^{\circ} \mathrm{C}$ sample temperature). Uncorrected data.

emission band as the pure LIF excitation spectrum of MHD, also because its maximum at $284 \mathrm{~nm}$ is red-shifted by about $20 \mathrm{~nm}$ with respect to its vapour phase absorption spectrum. From the extremely weak fluorescence observed for MMD, M4D and MHD under jet-conditions, one may conclude that $S_{1}-S_{2}$ coupling is extensive in these molecules, as to be expected on the basis of the close proximity of the two electronic states.

\subsection{Rotational contour analysis with DMABN}

At first glance, the spectrum of DMABN in Fig. 1 is an example of a relatively unperturbed LE-excitation spectrum at low excess energies above $E_{0}^{0}$. However, results to the contrary were obtained by Salgado et al. when they carried out a rotational contour analysis of several of the vibronic transitions in the spectrum over the excess energy range of $76-1158 \mathrm{~cm}^{-1}$ [11]. By fitting the experimental contours of their spectral lines and using calculated rotational constants, a rotational temperature of the ensemble of $5 \mathrm{~K}$ and the spec- 
tral resolution of their apparatus, they arrived at surprisingly large homogeneous rotational line widths in the range between 0.3 and $3.5 \mathrm{~cm}^{-1}$. These widths were interpreted as an indication for a fast intramolecular non-radiative process on a picosecond timescale (1.5-24 ps) starting from the Franck-Condon (FC) state, i.e., preceding fluorescence emission. According to this view, the LE state of DMABN is not the primarily emitting state under jet conditions. In support of this interpretation, indications of corresponding exponential rise components in the fluorescence emission traces of jet-cooled DMABN measured by time correlated single photon counting [8] were reported to be present in the original data [11].

Considering the low rotational temperature of $5 \mathrm{~K}$, the large spectral widths of the rotational contours observed for DMABN were puzzling [11]. The measured half-widths as taken from the published spectra gradually increase from 2.6 up to $4.2 \mathrm{~cm}^{-1}$ for transitions at 76 and $916 \mathrm{~cm}^{-1}$, respectively. A width of $3.4 \mathrm{~cm}^{-1}$ was observed for the transition at $136 \mathrm{~cm}^{-1}$, with a contour identified as C-type. This transition is somewhat broader than expected from the general trend, signalling a short lifetime of $6.5 \mathrm{ps}$ for the excited vibronic state. The line was assigned to an out-of-plane transition leading to a twisted geometry of the excited state. In contrast, a B-type rotational contour for this transition having a width of around $2 \mathrm{~cm}^{-1}$ may be estimated from the published spectra $[12,13]$.

In view of this discrepancy we took a closer look at some of the most prominent transitions of DMABN measured with our experimental setup (Fig. 1). Under optimal cooling conditions for isolated medium size molecules such as trans-stilbene, 1,4-diphenyl-(1,3)-butadiene, 4-dimethylamino-4'-cyanostilbene, azulene, phenylindene, and others (no dimers or higher oligomers detectable) we have consistently found rotational temperatures of about $8-10 \mathrm{~K}[14,15]$ so we would not expect this temperature to be significantly lower in our present jet expansion seeded with DMABN. All rotational contours we observe agree in shape with those published by Salgado et al. [11], except for the contour of the transition at $136 \mathrm{~cm}^{-1}$. For the latter transition we find, in agreement with $[12,13]$, a rotational contour indicating a B-type transition, see Fig. 5. In addition, the widths of the rotational contours of the low-energy transitions in our measurements are significantly smaller than those of Salgado et al., as illustrated in Fig. 5. Applying a rotational contour analysis to estimate the underlying homogeneous line-widths of the rovibronic transition yields Lorentzian widths
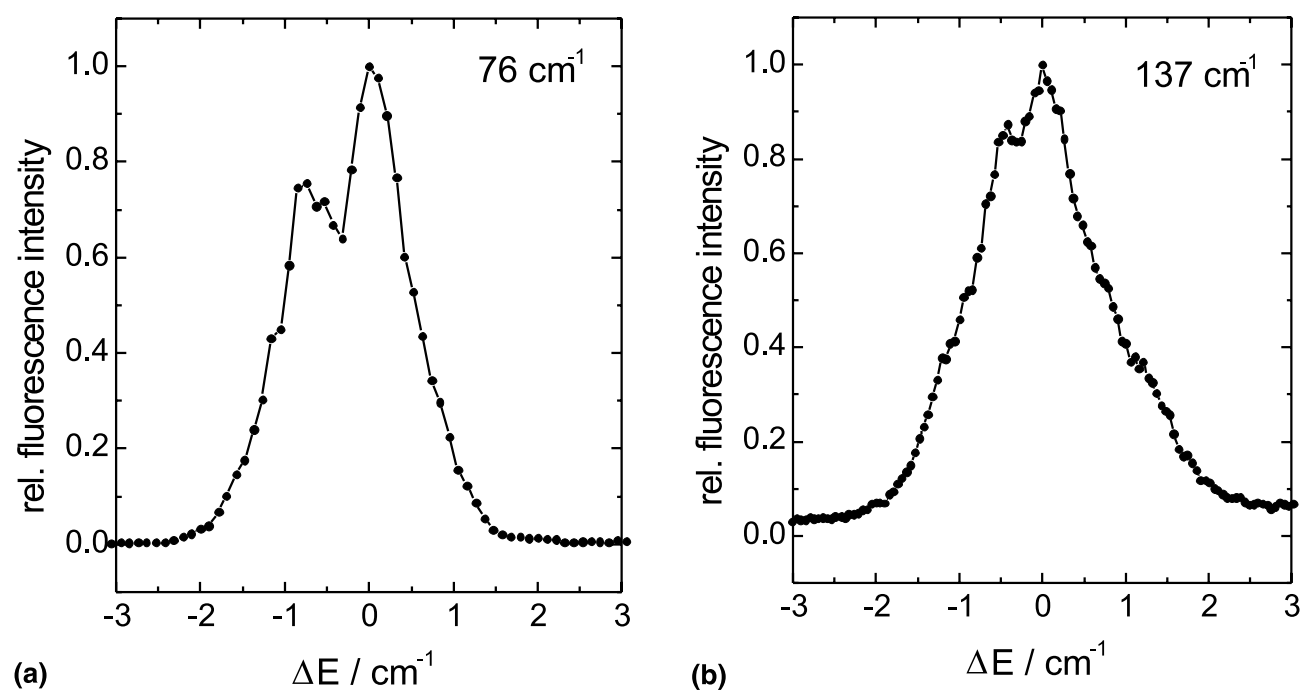

Fig. 5. Rotational contours of vibronic transitions of jet-cooled DMABN at: (a) $76 \mathrm{~cm}^{-1}$; (b) $137 \mathrm{~cm}^{-1}$. 
about an order of magnitude smaller than those given in [11], taking into account the presumably higher rotational temperature and the similar apparatus bandwidth of $0.17 \mathrm{~cm}^{-1}$ in our experiments. This implies significantly longer lifetimes of the LE state on the order of $100 \mathrm{ps}$ or more in the excess energy range up to $200 \mathrm{~cm}^{-1}$. These lifetimes should have been clearly visible as an initial rise in the corresponding time resolved fluorescence signals if the LE state was merely a precursor of the emitting state under jet conditions. In our view, the absence of a corresponding lifetime component as well as the fact that a B-type contour is observed for all low-energy transitions of jet-cooled DMABN cast considerable doubt on the interpretation of the line-widths in the spectrum presented in [11].

\section{Conclusion}

A comparison of the fluorescence excitation spectra of jet-cooled DIABN and DMABN shows that, in contrast to DMABN, the spectrum of jetcooled DIABN is characterized by an intense $0-0$ transition and a low frequency part consisting of many intense lines. The spectrum completely collapses above an excess excitation energy of $800 \mathrm{~cm}^{-1}$, indicating the presence of an efficient non-radiative decay channel related to rapid ICT in non-polar solution and DIABN vapour. For the compounds MHD, M4D, and MMD only extremely weak and structureless emission was obtained from correspondingly seeded jet-cooled expansions. In addition, a reinvestigation of the rotational contours of selected DMABN transitions did not reveal any indications of a fast nonradiative process at low excess energies.

\section{Acknowledgements}

Thanks are due to Dr. W. Kühnle and J. Bienert for synthesis and purification of the compounds. Generous financial support by the Deutsche Forschungsgemeinschaft (Sonderforschungsbereich 357, Molekulare Mechanismen unimolekularer Prozesse) and the Volkswagens- tiftung (Project: Intra- and Inter-molecular Electron Transfer).

\section{References}

[1] W. Rettig, B. Bliss, K. Dirnberger, Chem. Phys. Lett. 305 (1999) 8.

[2] K.A. Zachariasse, Chem. Phys. Lett. 320 (2000) 8.

[3] Z.R. Grabowski, K. Rotkiewicz, A. Siemiarczuk, D.J. Cowley, W. Baumann, Nouv. J. Chim. 3 (1979) 443.

[4] K.A. Zachariasse, M. Grobys, T.v.d. Haar, A. Hebecker, Yu.V. Il'ichev, Y.-B. Jiang, O. Morawski, W. Kühnle, J. Photochem. Photobiol. A 102 (1996) 59.

[5] K.A. Zachariasse, M. Grobys, Th. von der Haar, A. Hebecker, Yu.V. Il'ichev, O. Morawski, I. Rückert, W. Kühnle, J. Photochem. Photobiol. A 105 (1997) 373.

[6] A. Demeter, S. Druzhinin, M. George, E. Haselbach, J.-L. Roulin, K.A. Zachariasse, Chem. Phys. Lett. 323 (2000) 351.

[7] T. Kobayashi, M. Futakami, O. Kajimoto, Chem. Phys. Lett. 130 (1986) 63.

[8] E.M. Gibson, A.C. Jones, D. Phillips, Chem. Phys. Lett. 136 (1987) 454.

[9] R. Howell, H. Petek, D. Phillips, K. Yoshihara, Chem. Phys. Lett. 183 (1991) 249.

[10] R. Howell, D. Phillips, H. Petek, K. Yoshihara, Chem. Phys. 188 (1994) 303.

[11] F. Pérez Salgado, J. Herbich, A.G.M. Kunst, R.P.H. Rettschnick, J. Phys. Chem. A 103 (1999) 3184.

[12] B.D. Howells, J. McCombie, T.F. Palmer, J.P. Simons, A. Walters, J. Chem. Soc. Faraday Trans. 88 (1992) 2595.

[13] C. Dedonder-Lardeux, C. Jouvet, S. Martrenchard, D. Solgadi, J. McCombie, B.D. Howells, T.F. Palmer, A. Subaric-Leitis, C. Monte, W. Rettig, P. Zimmermann, Chem. Phys. 191 (1995) 271.

[14] R. Daum, T. Hansson, R. Nörenberg, D. Schwarzer, J. Schroeder, Chem. Phys. Lett. 246 (1995) 607.

[15] C. Lienau, J. Schroeder, J. Troe, K. Wack, Ber. Bunsenges. Phys. Chem. 101 (1997) 614.

[16] V.H. Grassian, J.A. Warren, E.R. Bernstein, H.V. Secor, J. Chem. Phys. 90 (1989) 3994.

[17] U. Lommatzsch, A. Gerlach, C. Lahmann, B. Brutschy, J. Phys. Chem. 102 (1998) 6421.

[18] R.A. Weersink, S.C. Wallace, R.D. Gordon, J. Chem. Phys. 103 (1995) 9530.

[19] U. Lommatzsch, B. Brutschy, Chem. Phys. 234 (1998) 35.

[20] G.D. Scholes, D. Phillips, I.R. Gould, Chem. Phys. Lett. 266 (1997) 521.

[21] G. Varsanyi, Assignment of vibrational spectra of seven hundred benzen derivatives, Akademiai Kiado, Budapest, 1974.

[22] L.W. Peng, M. Dantus, A.H. Zewail, K. Kemnitz, J.M. Hicks, K.B. Eisenthal, J. Phys. Chem. 91 (1987) 6162.

[23] T. Kobayashi, M. Futakami, O. Kajimoto, Chem. Phys. Lett. 141 (1987) 450. 